La détermination d'une ligne des neiges climatique - moyenne des versants exposés à l'«endroit» et à l'《envers» - est particulièrement difficile dans le domaine méditerranéen: en effet il n'y a pas de glaciers sur le versant sud. Nous ne pouvons pas non plus compenser cette absence par la différence de la ligne des neiges quaternaire. L'importance de l'exposition s'accrut lors du recul hors des régions plates de l'avant-pays dans les cirques escarpés et profonds. Sur le versant nord, des restes de glace et de névés surent se maintenir au pied des parois de cirques dans des situations spécialement favorables. Leur existence était particulièrement propice à l'origine de mouvements de masse de tous genres (p. ex.coulées de pierres). Les versants sud par contre furent entièrement déneigés, subirent une désagrégation régulière et intensive et une vaste couverture de débris s'étendit toujours plus haut jusqu'à la zone des sommets; ces faits ne soulignent pas seulement le contraste morphologique mais accentuent aussi la différence des lignes des neiges récentes entre les deux versants. Si nous obtenons une différence d'environ $200-300 \mathrm{~m}$ entre les versants nord et sud de l'Erciyas Dagh pour la glaciation quaternaire, cette différence s'élève à l'époque actuelle à au moins 40om; il en résulte une ligne des neiges climatique moyenne de tooom.

Lors d'autres parcours dans le Haut Bolkardagh du Taurus et dans le Kackar Dagh du Pont il fut possible de déterminer les lignes des neiges pour les régions de bordure: elles diffèrent fortement à cause du régime des précipitations varié. Dans le Taurus, le versant sud obtient plus de pluie; ainsi, la différence des lignes des neiges est quelque peu réduite (moyenne $3650 \mathrm{~m}$ ). Dans le Pont par contre, le versant nord obtient des précipitations de loin plus élevées (plus que $2400 \mathrm{~mm}$ ), si bien que la ligne des neiges s'élève très rapidement sur le versant sud sec et brûlant, c'est-à-dire de $3200 \mathrm{~m}$ sur le versant côtier à plus de $4000 \mathrm{~m}$ sur le versant à l'intérieur du continent.

La carte des lignes des neiges d'Anatolie montre le bombement de la surface délimitée par ces lignes à l'intérieur du continent, suivant ainsi la structure orographique et donc aussi climatique du pays. D'une part, c'est le régime des précipitations qui est déterminant, d'autre part, les isothermes d'été s'élèvent extraordinairement au-dessus de la surface chauffée d'Anatolie, si bien que cette élévation générale se montre aussi dans l'altitude de la ligne des neiges.

En définitive, la détermination d'une ligne des neiges récente dans les régions subtropiques et méditerranéennes est un problème difficile et les marges d'erreur seront toujours relativement hautes. L'existence de petits glaciers dans des situations locales particulièrement favorables nous induit toujours à nouveau à définir une ligne des neiges climatique trop basse; ainsi nous obtenons une moyenne de cotes bien trop basses pour la dépression de la ligne des neiges de la dernière glaciation quaternaire. D'autre part, l'étendue modeste et le fort recul des glaciers existants encore de nos jours indiquent qu'ils n'ont probablement pas survécu à la période de réchauffement postglaciaire et ne sont ainsi des témoins d'une ancienne époque glaciaire mais de nouvelles formations d'une époque d'extension récente.

\title{
KOSMISCHE STRUKTURPLÄNE DER TIBETER
}

\author{
SIEGBERT HUMMEL
}

In der völkerkundlichen Sammlung der Universität Zürich und in der Privatsammlung des Schweizer Geographen A. Dürst befinden sich je eine interessante Bronzetafel in Treibarbeit, die nur ganz gering in der mehr äußeren künstlerischen Durchgestaltung voneinander abweichen. Diese Platten sind ganz offensichtlich in Anlehnung an lamaistische Rollbilder (tib.: Thang-ka) geschaffen worden. ${ }^{1}$

Im Zentrum ist die Schildkröte (tib.: Rus-sbal) mit den schematisch dargestellten Ordnungsprinzipien und schöpferischen Funktionen unserer Welt. In den vier Füßen hält die Schildkröte gepfählte Frösche. Mit dieser Zeichnung wurde die altchinesische Tradition aufgenommen, wonach der Ahn der Chou-Dynastie, der König Wen, die im «Buche (aus dem Flusse) Lo», dem chinesischen Lo-Shu, niedergelegte Anordnung von acht Grundzeichen (chin.: Pa-Kua, tib.:sPar-kha) als Urzeichen für die Zustände des

1 Die Tafel der völkerk. Sammlg.: $35 \times 28,5 \mathrm{~cm}$; die in der Sammlg. Dürst: $29,2 \times 25,8 \mathrm{~cm}$. Vgl. d. lamaistische Thang-ka in Bronze auf Abb. S. 139 in E. Abegg, Die Indiensammlung der Universität Zürich, Zürich 1935; auch abgebildet bei Bl. Chr. Olschak, Religion und Kunst im alten Tibet, Zürich 1962, S.7o. Auf weitere Thang-kas mit den Themen der hier besprochenen Tafeln kommen wir noch zu sprechen (vgl. Anm. 4 und 13). 
werdehaften Seins aufgegriffen und diese mit divinatorischen Ratschlägen kommentiert habe. Bekanntlich wurde das die Grundlage für das berühmte chinesische «Buch der Wandlungen» (I-Ching).2 Dieses Schema ist von den Chinesen mit einer Schildkröte in Verbindung gebracht worden, während eine andere Anordnung ein Drache des HuangHo auf seinem Rücken gehabt haben soll. Wir finden unser Schema als acht Trigramme aus je drei übereinandergeschichteten, gebrochenen oder ungebrochenen horizontalen Strichen kombiniert, chinesisch als $\mathrm{Pa}-\mathrm{Kua}$ bezeichnet, auf dem Bauche der Schildkröte in einer Blüte kranzförmig um ein Zentrum gruppiert, das in neun Felder eingeteilt ist. Dieses magische Diagramm aus den sog. neun $s \mathrm{Me}[\mathrm{rMe}]-b a$ (= Flecken; tib.: sMeba-dgu) ist das fundamentale kosmische Kraftfeld, dessen neun Teile mit Zahlen versehen sind, die, aus jeder Richtung addiert, stets die Summe 15 ergeben. Das ist der statische Aspekt der in Gestalt von acht Trigrammen dynamischen Grundprinzipien. Jedes Glied des Feldes dient nach einer dem Hauptbegründer des Lamaismus, Padmasambhava (8. Jh.), zuge-chriebenen tibetischen Schrift, die der Errichtung magischer Dämonenfallen (tib.: mDos) gewidmet ist, ${ }^{3}$ einem $1 \mathrm{Ha}$, einer personifizierten kosmischen Mächtigkeit, als Aufenthaltsort. Das gilt auch von den Pa-Kua und den noch zu besprechenden Gliedern des Tierkreises. Jedes Feld hat eine bestimmte Farbe, die bei E. Schlagintweit (Le Bouddhisme au Tibet, Paris 1881, Tafel neben S. 192) angegeben und auf einem tibetischen Rollbild, das R. Bleichsteiner veröffentlicht hat, zu erkennen sind. Mit dieser Malerei stimmen nach Inhalt und Gliederung unsere Tafeln weithin überein. ${ }^{4}$ Man hat in das genannte Kraftfeld auch die fünf Elemente chinesischer Tradition einzubauen versucht, die ebenfalls als $1 \mathrm{Ha}$ auftreten. So werden in der genannten tibetischen Schrift die Elemente als dem Bereich der großen goldenen Schildkröte zugehörig verstanden (tib.:Mahâ-gser-gyi-rus-sbal-srid). Nach der seit der Han-Zeit in China üblichen Farbgebung für die Elemente deuten die weißen Felder 8, 1, 6 (unten von links nach rechts) auf das Metall bzw.das Eisen (tib.: lCags), die blauen bzw. schwarzen 2 und 3 (oben rechts und Mitte links) auf das Wasser (tib.: Chu), das grüne Feld 4 (oben links) auf das Holz (tib.: Shing), das gelbe (5, Mittelpunkt des Diagrammes) auf die Erde (tib.: Sa) und die roten Felder 7 und 9 (Mitte rechts und oben Mitte) auf das Feuer (tib.: Me). Nach der Lehre von den Entsprechungen stehen die genannten Elemente, und damit auch die sMe-ba, wieder mit dem menschlichen Organismus als Mikrokosmos in Beziehung, und zwar nach tibetischer Vorstellung die Erde mit dem Fleisch, das Metall mit den Knochen, das Feuer mit der Körperwärme, das Wasser mit dem Blut und die Luft bzw. der Wind, der seit der Han-Zeit dem Holz gleichgeordnet ist, mit dem Atem (1.c., Fol. 3b/4a). Diese Entsprechungen weichen damit von den in China üblichen etwas ab. ${ }^{5}$

Um die $\mathrm{Pa}-\mathrm{Kua}$ ist auf der Schildkröte als weiterer kosmischer Ordnungsfaktor der kleine Jahreskreis (tib.: Lo-'khor) gelegt. Er besteht aus Maus (tib.: Byi-ba), Tiger (sTag), Drache ('Brug), Pferd (rTa), Affe (sPre['u]), Hund (Khyi), Ochse (Glang), Hase (Yos), Schlange (sBrul), Schaf (Lug), Vogel (Bya) und Schwein (Phag). Dieser Tierkreis soll zur Ordnung der Jahre in einem mit der Maus beginnenden Zwölferzyklus den Tibetern schon unter König Srong-btsan-sgam-po ( $\dagger$ um 650) durch des:en chinesische Gemahlin bekannt geworden sein. Im Jahre 1027 wurde dann mit Einführung des Kâlacakra-Systems (tib.: Dus-kyi-khor-lo) dieser Zwölfjahrzyklus durch Kombination mit den Elementen als Sechzigjahrzyklus allgemein üblich

2 Vgl. I Ching, das Buch der Wandlungen, ed. R. Wilhelm, Jena o. J.-J. Schubert, Tibetische Äquivalente der I-ching- Hexagramme (in: Mitt. des Inst. f. Orientforschung, IV, 1956, S. 411 ff.).

3 Slob-dpon-pad-ma-'byung-gnas-kyis-mdzad-pai-mdos-dgu-pa-zhes-bya-ba-bzhugs-so für Völkerkunde, Leipzig, Sign. F III, 11).

4 Hochasien (in: H. A. Bernatzik, Die Große Völkerkunde, Bd. II, Leipzig 1939, Farbtafel neben S. 80).

5 Vgl. S. Hummel, Lamaistische Studien, Leipzig 195o, S .76, mit dem Wortlaut der erwähnten tibetischen Schrift (Fol. 3b/4a). 


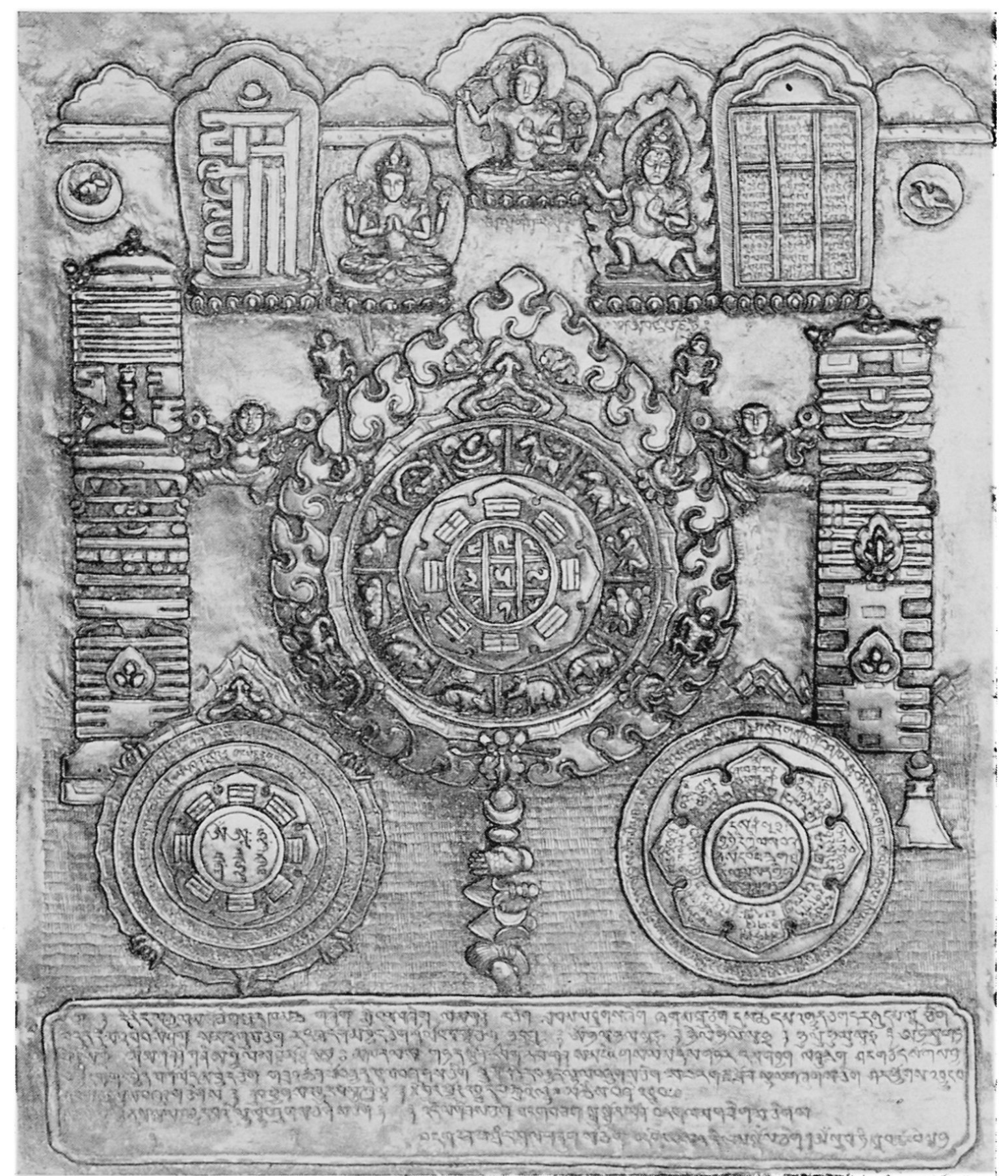

Tibetische Bronzetafel (35 x 28,5 cm). Sammlung für Völkerkunde der Universität Zürich, Kat.-Nr. 12664

(tib.: Lo-khams). Dieses System ist auch als Rab-byung bekannt, genannt nach dem ersten Jahre seiner Einführung in Übersetzung der indischen Bezeichnung jenes Jahres als Prabhava (tib.: Rab-byung). Auf die Übereinstimmung des chinesisch-tibetischen Tierkreises mit dem persischen, während der indische mehr dem alt-vorderorientalischengriechischen gleicht, hat M. Hermanns hingewiesen. ${ }^{6}$

6 Zum Problem der Jahreszyklen in Tibet: B. Aoki, Study on Early Tibetan Chronicles, Tokyo 1955, S. 11 ff. - P. Poucha, Innerasiatische Chronologie (Mongolische Miszellen VII, in: Central Asiatic Journal, VII, 3). - M. Hermanns, Mythen und Mysterien der Tibeter, Köln 1956, S. 44 ff. 


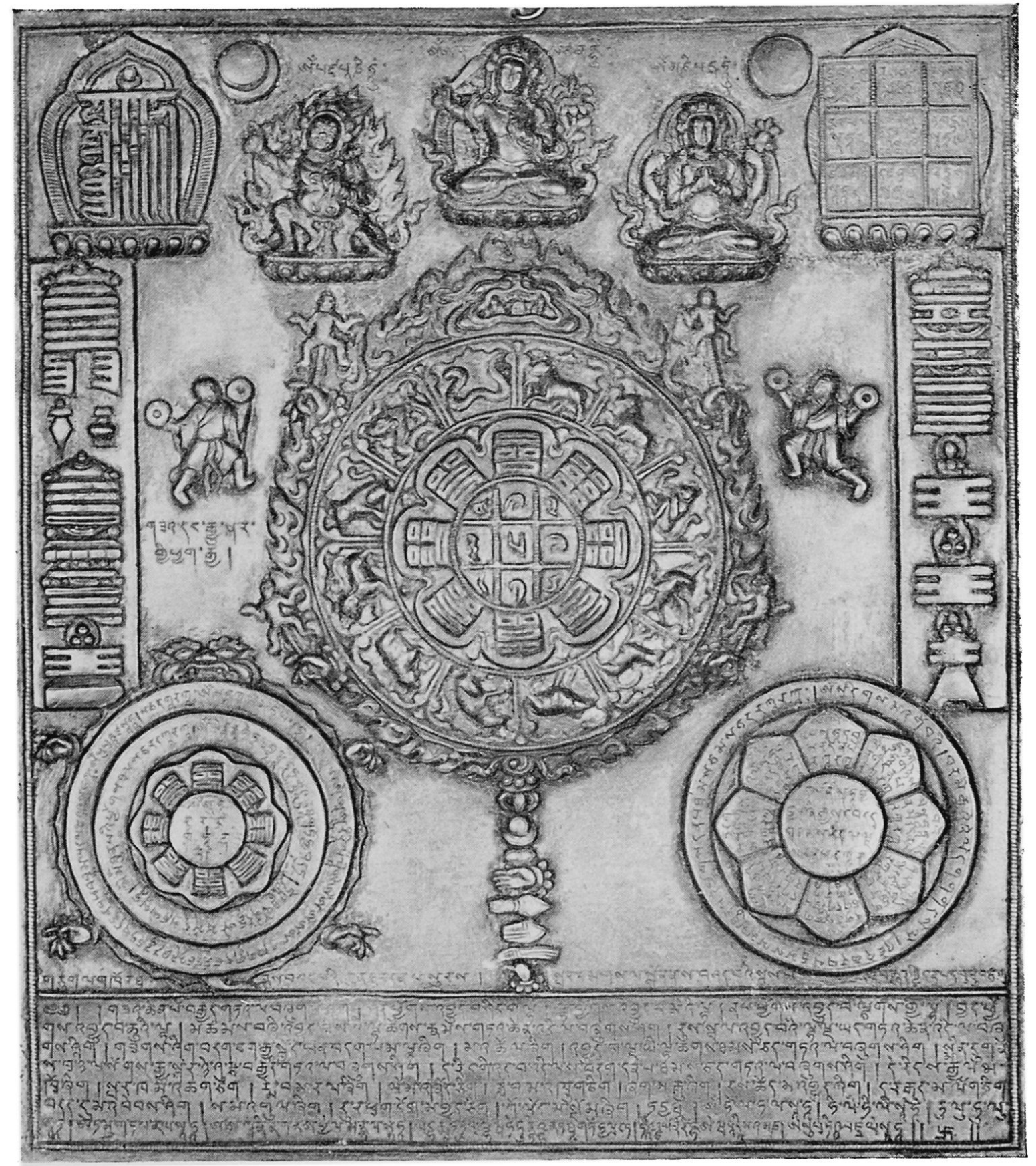

Tibetische Bronzetafel $(29,2 \times 25,8 \mathrm{~cm})$

`Sammlung A. Dürst

Unter der Schildkröte finden sich übereinandergeordnet die Zeichen der Planeten bzw. der Wochentage. Die sieben Planeten Sonne, Mond, Merkur, Venus, Mars, Jupiter und Saturn werden oft durch ein achtes Zeichen, den aufsteigenden Knoten, erweitert und sind dann als gZa'-brgyad bekannt. Manchmal kommt noch der absteigende Knoten als neuntes Zeichen hinzu. Die Wochentage erkennt man, in der Reihe von oben nach unten, an einem Ball (auf Malereien $\operatorname{rot}^{7}$ ) für den Sonntag (tib.: gZa'-nyi$\mathrm{ma})$, einer blauen Mondsichel für den Montag ( $\mathrm{gZa} \mathrm{a}^{\prime}$-zla-ba) einem roten Auge für den

7 Die Farbangaben sind den entsprechenden Malereien entnommen; vgl. auch L. A. Waddell, The Buddhism of Tibet, London 1895, S. 455 (das Exemplar dieses Werkes in der Bibliothek des Verfassers wurde durch S. H. Ribbach, früher in Leh, koloriert). 
Dienstag (gZa'-mig-dmar), einer blauen Hand für den Mittwoch (gZa'-lhag-pa = Merkur), einem blauen Ritualdolch für den Donnerstag ( $g Z a^{\prime}$-phur-bu), einem gelben Band für den Freitag (gZa'-pa-sangs) und einem gelben Bündel für den Sonnabend (gZa'-spen-pa = Saturn).

Neben der Schildkröte, links und rechts, vertreten zwei Gestalten die achtundzwanzig als weibliche Gottheiten gedachten Mondstationen (tib.: rGyu-:kar), d.h. Konstellationen von Sternen, die der Mond bei seinem Umlauf durchläuft. Da diese Stationen auch mit Tiernamen bezeichnet werden, bilden sie eine Art Mondtierkreis. ${ }^{8}$

Über der Schildkröte sieht man die beliebte lamaistische Trinität aus Mañjushrî bzw. Mañjughosha, Padmapâni und Vajrapâni ${ }^{9}$ mit ihrem Mantras. Sie sind auch als Rig'dzin-mgon-po bekannt und mitunter durch drei Pagoden (tib.: mChod-rten) angedeutet, die den drei Bodhisatvas entsprechend gelb oder rot, weiß und blau bemalt werden. Diese Pagoden bringt der Tibeter oft mit vorbuddhistischen Vorstellungen von den drei Stockwerken (tib.: sTong-gsum) eines trichotomischen Weltbildes in Zusammenhang. Da Mañjushrî (Mañjughosha), in der Mitte der Trinität, nach der lamaistischen Überlieferung an der Weltschöpfung beteiligt war, indem er eine Schildkröte bei der Überwindung des Chaos als Basis für den Kosmos schuf (Schlagintweit, l.c. S. 200), hat er bzw. seine Trinität einen sinnvollen Platz in dem ko mischen Gefüge der Tafel. Es ist dann auch verständlich, warum. Mañjushrî zum Schutzherrn der Astrologen (tib.: rTsis-pa) geworden ist.

Links von den drei Bodhisatvas steht in einer Lotusblüte das berühmte Diagramm rNam-bcu-dbang-ldan ( $\mathrm{kr}$.: Dashâkâro-vashî), das mit dem synkretistischen, stark iranisch beeinflußten System Kâlacakra verbreitet worden ist. ${ }^{10}$ Dieses Diagramm stellt die gegliederte Ganzheit des Mikro-Makrokosmos und damit auch des psychosomatischen Organismus des Menschen dar. ${ }^{10}$

Recht; von den Bodhisatvas, deren Mantras bzw. Anrufungsformeln unter den Gottheiten zu lesen sind, ist in einer Lotusblüte die Tafel der sMe-ba errichtet, die auf den Malereien ebenfalls, entsprechend dem farbigen Feld der Schildkröte, koloriert sind. In die Felder sind neben mystischen Formeln (skr.: Dhâranî) die auf die Farben hinweisenden Zahlen eingetragen. Diese Tafel in der Lotusblüte wird nach der Inschrift des Stückes in der Sammlung Dürst auf Padmasambhava als ihren Erfinder zurückgeführt und als wirkungskräftig gegen allerlei Übel bezeichnet (tib.: ngan-pa-guthub $=$ abwehrkräftig). Dieses Diagramm auf der Lotusblüte stehend, ist demnach eine

8 In A. K. Gordon, Tibetan Religious Art, New York 1952, Farbbild S. 27 mit einer Malerei ähnlich unserer Bronzeplatte, sind die 28 Mondstationen als weibliche Gottheiten vollständig dargestellt. Sie nehmen dort den gleichen Ort ein wie auf unserer Tafel. Man findet diese Nakshatras (skr.) auch auf Tafel 2 in: A. Grünwedel, Der Weg nach Sambhala, München 1915.

9 Vgl A. H. Francke, Tibetische Hochzeitslieder, Hagen und Darmstadt 1923, Tafel 2 und 4. - S. Hummel, Euras. Traditionen in der tibet. Bon-Religion (in: Opusc. Ethn. Mem. Lud. Biro Sacra, Budapest 1959, S. 178). Für Rig-dzin-mgon-po (Francke, 1. c.) findet sich auch Rigs-gsum-mgon-po, d. h. Vorsteher der wichtigsten drei Familien von Gottheiten. Diese Familien (skr.: Kula) sind die Tathâgata-Familie mit Shakyamuni und Mañjushrî als Bodhisatva, die PadmaFamilie mit Amitâbha und Avalokiteshvara, die Vajra-Familie mit Akshobhya und Vajrapâni. So ist d. Mañjushrîmûlakalpa-tantra, jenes Werk, das im 8.-10. Jh. abschließend redigiert wurde, der Tathâgata-Familie gewidmet. Nach populären Vorstellungen werden wegen ihrer Farbe mit der roten Pagode auch die bTsan-Dämonen und mit der schwarzen die schwarzen bDud in Verbindung gebracht, obwohl die bDud zu den Dämonen der Luft gehören (vgl. H. Kihara, Peoples of Nepal Himalaya, Kyoto 1957, S. 146-160: Tibeter in Nord-Nepâl). Zu den gen. Familien von Gottheiten vgl. A. Macdonald, Le Mandala du Mañjusrîmûlakalpa, Paris 1962, Einleitung (Rezens. S. Hummel, in: Tribus, Bd. 12); ferner D. L. Snellgrove, Buddhist Himâlaya, Oxford 1957, S. $62 \mathrm{f}$., $75 \mathrm{ff}$. (für Shakyamuni später auch Vairocana). Zu den Mantras vgl. L. A. Waddell, 1, c., S. 15o f. - Tafel von Dürr: Formel f. Mañjughosha; Tafel ds Mus.: Formel f. Mañjushrî, einer Abart des Mañjughosha (vgl. d. einschlägigen Ikonographien).

10 Zum Kâlacakra-System finden sich ausführliche Hinweise in J. Schubert. Das Wunschgebet um Sambhala (in: Mitt. des Inst. für Orientforschung, I/3, S. 424 ff.). 
durch Padmasambhava eingeführte Abwandlung des längst bekannten Schemas der Neun Häuser, der sMe-ba-dgu (wörtlich: Neun Flecken). Links und rechts außen schweben ganz oben auf den Platten wie auf den gemalten Rollbildern Sonne und Mond. Die Sonne trägt den Sonnenvogel wie schon auf buddhistischen Malereien des 10. Jh. in Tun-Huang. Der Mond ist vom Mondhasen bewohnt, einer uralten über Eurasien verbreiteten Vorstellung gemäß, weswegen der Hase in Tibet noch bis in die Gegenwart hinein größte Verehrung genoß.11

Links unten auf beiden Platten ist nochmals die Schildkröte mit den aus dem Gesamtschema des Zentrums herausgelösten und auf die Blütenblätter einer Lotusblume gezeichneten Pa-Kua auf ihrem Bauche dargestellt. Das ist das Siegel des Anführers der Sa-bdag. Gegenüber sind die Blütenblätter in den Farben der Elemente bzw. der sMeba gezeigt. Beide Diagramme sind nach den Aufschriften der Malerei in Wien ebenfalls abwehrkräftig. Das zuletzt genannte schützt gegen die Râksha-Dämonen.

Seltsam sind zwei diagrammartige breite Bänder, die links und rechts von der zentralen Schildkröte vertikal verlaufen. Wir finden sie wieder bei L. A. Waddell, 1. c., S. 395. Dabei handelt es sich zum größten Teil um spezifisch tibetische Neuschöpfungen in Anlehnung an die bekannten chinesischen $\mathrm{Pa}-\mathrm{Kua}$, aber in völligem Mißverständnis des diesen zugrunde liegenden Prinzips. Die Pa-Kua sind offensichtlich nur als magische Zeichen aufgefaßt worden. Schlagintweit (1.c., S. 203) hat einige weitere Beicpiele gegeben, bei denen die Verwendung von 4-6 horizontalen und mitunter auch verbindenden vertikalen Strichen auffällt. ${ }^{12}$ Diese tibetischen Erfindungen gelten als besonders wirksam gegen dämonische, das kosmische Gefüge und den menschlichen Organismus gefährdende Einflüsse und sind keine horoskopartigen Niederschriften. Sie haben auf un eren Tafeln ihren wohldurchdachten Ort. Das linke Band besteht aus zwei, je von einem Baldachin gekrönten, übereinander geordneten Konstellationen, die den $\mathrm{lHa}$ des Himmelsraumes gehören, während sich das rechte Band auf die Gottheiten des irdischen Bereiches bezieht. Es handelt sich jedesmal um eine Herrschaftsbekundung bzw. um die Bekräftigung (Phyag-rgya) der Gegenwart der betr. 1 Ha. Auf dem durch R. Bleichsteiner in A. Bernatzik, 1. c., veröffentlichten Rollbild (Mus. f. Völkerkunde Wien, Inv. Nr. 91933 ; vgl. Anm. 4), von dem ich Herrn Dr. A. Janata vom Museum f. Völkerkunde in Wien ein gutes Photo verdanke, sind die gleichen Bänder in der selben Konstellation, die auch genau denen bei L. A. Waddell, S. 355 entsprechen, bedeutsamerweise tibetisch beschriftet. Links oben liest man «Siegel des Zwölfjahreskreises», links unten «Siegel der Planeten und Mondstationen», rechts «Siegel der Klu, gNyan und Sa-bdag», wobei «Klu-gnyan-sa-bdag-gi-phyag-rgya» für «Klu-gnyen-sa-bdag-giphyag-rgya»zu lesen sein wird, da Klu (= Nâgas), gNyan und Sa-bdag als Geister der sog. Mittelwelt in den Texten, vornehmlich der Bon-Religion, gern zusammen auftreten, wenn auch die einzelnen Herrschaftsbereiche oft nicht scharf getrennt sind und sich überschneiden; so bewohnen z.B. die Klu auch Bäume und Felsen. Wo jedoch die drei Gruppen zusammen genannt werden, wird man den Klu die Gewässer und den gNyan die Berggefilde, Holz und Stein zusprechen, wogegen die Sa-bdag dann die eigentlichen Erdgeister sind.

Die einzelnen Bildglieder der Bronzeplatten mußten hier nach ihren bekannten Einzelheiten unter besonderen Gesichtspunkten nochmals skizziert werden, weil Darstellungen dieser Art in der einschlägigen Literatur einfach als Divinationstafeln, Kalender oder astrologische Schemata bzw. Horoskope bezeichnet werden, was aber völlig falsch ist. Die tibetischen Astrologen bzw. die Wahrsager bedienen sich in ihrer Praxis nicht dieser stereotyp gestalteten Darstellungen,

11 Vgl. F. Grenard, I. L. Dutreuil de Rhins, Miss. Scient. dans la Haute Asie, Vol. II, Paris 1898, S. 403. Für China vgl. u. a. C. Hentze, Die Sakralbronzen und ihre Bedeutung in den frühchinesischen Kulturen, Antwerpen 1941, Bd. I, S. 73, 139, 169, 190.

12 Vgl. auch A. K. Gordon, Tibetan Religious Art, New York 1952, Farbtafel S. 27 mit einem unseren Platten verwandten Rollbild. Eine weitere sehr ähnliche Malerei zeigt auch R. Bleichsteiner, Die Gelbe Kirche, Wien 1937, Abb. 72. 
sondern benutzen weit kompliziertere Hilfsmittel.13 Aus unserer Beschreibung der verschiedenen Diagramme auf den beiden Bronzeplatten wie auf den verwandten Rollbildern wird es deutlich geworden sein, daß derartige Darstellungen dic segensreichen Kraftfelder, Ordnungsprinzipien und Funktionsgrundlagen im Kosmos und einige Zeichen im Dienste der Aktivierung oder des Schutzes dieser Mächtigkeiten schematisch in sich vereinigen und magisch entfalten sollen. Diese Deutung wird auch nicht dadurch entkräftet, daß die verschiedenen Schemata bei der Divination eine Rolle spielen und in den dazu benutzten Handbüchern oder Plänen vorhanden sind. Die Aufgabe dieser gemalten oder in Metall hergestellten Tafeln ist die gleiche wie die von ikonographischen Thang-kas, insbesondere aber von solchen, auf denen mit Hilfe von lamaistischen Gottheiten, deren Attributen oder Keimsilben Mandalas (tib.: dKyil-'khor), d.h. fundamentale Schemata von Seinszusammenhängen, abgebildet sind.

Die neun sMe-ba gelten, wie schon gesagt, als Wohnungen von $1 \mathrm{Ha}$, besser vielleicht als Gegenwartshindeutungen dieser $1 \mathrm{Ha}$, wobei die diesbezüglichen Vorstellungen schillernd ineinander übergehen. Unter Verwendung vorbuddhistisch-tibetischer Überlieferungen werden diese $1 \mathrm{Ha}$ als Sa-bdag (genii loci) bezeichnet. Ihre Namen sind nach R. de Nebesky-Wojkowitz 14 Sai-lhamo Rab-brtan-ma (Feld I), bDud-gyi-rgyal-po (Feld 2), Sa-bsen-gdug-byed (Feld 3), 1Ha-modal-byad bzw. Klu-rgyal-lba-ru (Feld 4), Sa-bdag-rgyal-po-'jig-rten-bdag (Feld 5), rGyal-poskye-'groi-dpal (Feld 6), bTsan-dmar-chen-po (Feld 7), lHa-chen-dbang-phyug-che (Feld 8) und Ma-mo-dza-mun-ti (Feld 9). Das für die sMe-ba-dgu Gesagte trifft auch für die übrigen Gruppen zu. So fungieren auch die Pa-Kua als Residenzen von $1 \mathrm{Ha}$ im Sinne von Sa-bdag. Ihre ikonographische Gestalt hat A. Grünwedel bekannt gemacht.15 Ihre tibetischen Namen sind nach der Ordnung von König Wen, der Einfachheit halber im Diagramm der Schildkröte oben Mitte beginnend nach rechts herum, Li (chin.: Li), Khon (chin.: K'un), Dwal (chin.: Tui), Khen (chin.: Ch'ien), Kham (chin.: K'an), Gin (chin.: Ken), Zin (chin.: Chen) und Zon (chin.: Sun). Auf unseren Tafeln sind jedoch Vertauschungen vorgenommen worden.16 Die Frage, ob in diesen Veränderungen der chinesischen traditionellen Ordnung auf den tibetischen Darstellungen Fehler oder besondere magische Absichten vorliegen, muß hier offenbleiben.

Wie zwischen den $\mathrm{Pa}-\mathrm{Kua}$, den sMe-ba und den Elementen bzw. dem menschlichen Organismus, so besteht auch zwischen den Elementen und den Planeten eine Verbindung. Zum Wasser gehört Merkur, zum Metall Venus, zum Feuer Mars, zum Holz Jupiter und zur Erde Saturn. Diese Elementen-Planetengleichungen sind in China zur Han-Zeit nachweisbar.17 Die gesamte Tafel stellt somit einen fein gegliederten kosmischen Organismus dar.

Von allen Gruppen gilt nun entsprechend dem Wesen der Sa-bdag, daß sie dem Menschen auch gefährlich werden können. In der dem Padmasambhava zugeschriebenen Anweisung (1.c., Fol. 3a) heißt es stets, die einzelnen betreffenden $1 \mathrm{Ha}$ möchten nicht in bedrohliche Erregung geraten (tib.: ma-'khrugs-cig).

Wie bei den mDos werden auch in den Schemata unserer Tafeln die verschiedenen Mächtigkeiten in ihrer harmonischen Ordnung festgehalten, lokalisiert. im Ritus genährt, an gefährlicher Verselbständigung oder daraus entstehender Hypertrophie, d. h. an der Erregung, gehindert und nur in solcher Kontrolle segensreich, wie das in gleicher Weise von verschiedenen Angehörigen des lamaistischen Pantheons gilt. Ähnlich den mDos dienen die Schemata der Abwehr und heilsamen Aktivierung zugleich. Darin erweisen sie sich eben vielen lamaistischen Mandalas im Bereiche der Ikonographie als verwandt.

Die besonders enge Verbindung in dem Gefüge der Tafeln zwischen den Pa-Kua und den sMeba-dgu ist nicht eine tibetische Konstruktion, sondern beruht auf der eingangs erwähnten chinesischen Tradition, wonach die Neun sMe-ba, die in der chinesischen Divinationspraxis zur HanZeit auf Grund älterer Traditionen als die Chiu-Kung (= Neun Hallen) bekannt waren, mit jenem Lo-Shu in Zusammenhang gebracht wurden. Sie sollen auf dem Panzer der Schildkröte in

13 Vgl. A. K. Gordon, 1. c., Abb.auf S. 26 mit schönen Beispielen. Ferner R. v.Nebesky-Wojkowitz, Wo Berge Götter sind, Stuttgart 1955, Abb. neben S. 16o. Wahrsagebücher (tib. rTsisdpe) und Kalender (tib.: Zla-tho) zeigt auch H. Kihara, 1. c., S. 193. - Das bei Gordon, 1. c., S. 89 oben abgebildete Motiv auf einer Amulettkapsel (tib.: Ga'u) ist darum auch kein Horoskop, sondern ganz allgemein gehalten und hat lediglich apotropäische Bedeutung. Horoskope sind individuell verschieden. In gleicher Weise entbehren unsere Bronzetafeln jeden individuellen Zuschnitt.

14 Oracles and Demons of Tibet, 's-Gravenhage 1956, S. 294.

15 Die Sternschnuppen im Vaidûrya-dkar-po (in : Festschrift Eduard Seler, Stuttgart 1922, Fig. 4).

16 Auch dann, wenn die Tafeln in der nach J. Schubert, 1. c., vorgeschlagenen Weise gedreht werden.

17 Vgl. hierzu wie auch zu der bereits genannten Gleichsetzung Elemente-Organismus des Menschen W. Eberhard, Beiträge zur kosmologischen Spekulation Chinas in der Han-Zeit (in: Baessler-Archiv 1933). 
Form von Flecken zu sehen gewesen sein 18 und die Art der Anordnung der Pa-Kua durch König Wen als Zeichen für das wandelhafte Sein bestimmt haben. Nach Schuyler Cammann sind die Chinesen wahrscheinlich überhaupt in einem weiten Umkreis Eurasiens die Erfinder der magischen Quadrate und ihres Gebrauchs als wirksame Mittel der Abwehr oder zur Entwicklung guter Kräfte. Im mDzad-pai-kun-rdzob-gya-sel-me-long des Chags-med-rin-po-che, Fol. 12b15a, heißt es folgerichtig, daß man mit einer Zeichnung der Neun-sMe-ba inmitten der Acht-sParkha alle Arten von Unheil unter Kontrolle bringt.19 Der besseren Vergegenwärtigung aller in den Tafeln verzeichneten Kräfte dient dann die Eintragung der magischen Silben (skr.:Dhâranî) in die zuständigen Häuser.

Schon im Shu-Ching, das nach W. Eberhard etwa im 7. Jh. v. Chr. niedergeschrieben worden ist, 20 wird erwähnt, daß man Schildpatt erhitzte und die dadurch entstandenen Risse divinatorisch ausdeutete. Es ist also nicht verwunderlich, wenn die sMe-ba, ursprünglich wohl die Häuser der Planeten,21 der chinesischen Tradition gemäß mit den Pa-Kua auch auf unseren Bronzeplatten eine bevorzugte Rolle spielen und daß sie in der wenig bekannten tibetischen Chiromantie befragt wurden, 22 weisen sie doch samt ihrer planetarischen Beziehung auf die Struktur des psycho-somatischen Organismus. Lediglich diese Strukturverhältnisse des Mikro-Makrokosmos zu zeigen und zu aktivieren ist jedoch die Aufgabe der hier besprochenen Darstellungen.23

Die Inschriften der Diagramme mit den sMe-ba und den sPar-kha, unübersetzbare Dhâranîs und Mantras, bestätigen, daß wir es bei den Platten und den verwandten Malereien nicht mit Divinationstafeln, Horoskopen, astrologischen Schemata oder Kalendern zu tun haben. Vor allem gibt die Beschwörung am Fuße der beiden Bronzetafeln unserer Deutung recht. Darin werden die IHa und die Sa-bdag der verschiedenen Diagramme gebeten, ganz wie bei den mos der genannten Schrift des Padmasambhava (gl. Anm. 3 und 5), Wohnung zu nehmen, wie überhaupt diese Beschwörung einer kurzen Zusammenfassung der Hauptanliegen jener Schrift gleichkommt. Das Gefüge aus den $\mathrm{Pa}-\mathrm{Kua}$, sMe-ba sowie der verschiedenen Zeiteinteilungen vom Jahr bis zu den Stunden sowie der Rhythmus des Kosmos, so heißt es weiter, möchte in harmonischem Einklang bleiben und kosmische Katastrophen vermieden werden.

So dürfte es angebracht cein, Darstellungen wie die hier besprochenen künftighin als kosmische Strukturpläne zu bezeichnen. Ihre Aufgabe hatten wir skizziert.

\section{CALENDRIERS ASTROLOGIQUES DU TIBET}

Plusieurs images qui jouent un rôle important dans le lamaisme, figurent dans notre littérature sous de fausses désignations. Parmi celles-ci, il y en a un groupe de descriptions schématiques qui sont citées sous un titre bien défini. Il s'agit de réproductions connues - diagrammes astrologiques, respectivement calendriers astrologiques — dans le style des images roulées lamaistiques qui existent aussi sous forme de plaques métalliques ciselées. Dans la collection de l'institut d'ethnographie de l'université de Zurich se trouve un exemplaire de ces images roulées. L'observation de ces images et de ces inscriptions stéréotypées pour toutes les pièces ont montré une coordination des champs dynamiques et des fonctions de base qui ont la plus grande importance pour tout le cosmos. Leur accord et leur rythme préservent le monde et l'homme des catastrophes qui peuvent toujours survenir par la séparation de quelques membres d'une unité. Ces membres sont les octo-triagrammes ( $\mathrm{Pa}-\mathrm{Kua}$ ) et le carré magique des neuf halles (Chiu-Kung) qui dérivent de la cosmologie chinoise. Ils se trouvent placés sur le dos d'une torture et représentent la base mythologique du cosmos. Autres éléments du cosmos: le cycle annuel (Tib.: Lokhar), le zodiaque

$18 \mathrm{Vgl}$. die Zeichnung im I-Ching, ed. R. Wilhelm, S. 235, in Kombination mit den Pa-Kua. Schuyler Cammann, The Evolution of Magic Squares in China (in: Journal of the American Oriental Society, Vol. 8o, 2, S. 117). - Id., Old Chinese Magic Squares (in: Sinologica, VII, 1, S. $14 \mathrm{ff}$.).

19 Vgl. S. Hummel, Günstige und ungünstige Zeiten und Zeichen nach Chags-med-rin-po-che (in: Folklore Studies, Tokyo 1964). Auch das Lindenmuseum besitzt einige Amulette dieser Art (Nr. 72472.72474).

20 Geschichte Chinas seit der Han-Zeit (in: Historia Mundi, Bern und München, Bd. II, S. 565 ; das I-Ching wird darin ins 11. Jh. v. Chr. verwiesen). Nach H. Hackmann, Chinas Philosophie, München 1927, S. 28, dürfte das Schildkrötenorakel auch wegen des hohen Alters und der davon hergeleiteten ungewöhnlichen Erfahrung und Einsicht beliebt gewesen sein, wie man in China der Schildkröte nachsagte.

21 Einschließlich auf- und absteigenden Knoten.

22 B. Kellermann, Meine Reisen in Asien, Berlin 1940, S. 148 f.

23 Nach Schuyler Cammann (vgl. Anm. 18) sahen die Chinesen in dem Zahlendiagramm des Lo-Shu die kosmische Polarität, den Rhythmus der Jahreszeiten, die Fünf Elemente und die kosmischen Richtungen wie in einem mikro-makrokosmischen Modell des Universums vergegenwärtigt. 
de la lune, les planètes et les jours de la semaine qui ont connus pour l'organisme psycho-somatique comme diagramme sous le nom rHam-bcu-dbang-ldan du système de Kâlacakra. En outre les sceaux, respectivement les a misons des fantômes importants ( $T$ ib.: $1 \mathrm{Ha}$ ) de notre sphère se montrent influents comme signe de souveraineté. Ce système des images se place au service d'une connaissance pour un ordre prospère, pour un maintien et pour une activation du jeu harmonieux du dynamisme cosmique. Qu'il me soit permis de souligner que ces descriptions sont à interpréter dorénavant comme plans d'une structure cosmique.

\title{
ÜBER ÄQUIDEFORMATEN
}

\author{
J. BOLLIGER \\ 1. AEQUIDEFORMATEN \\ zu Albers flächentreuer Kegelprojektion
}

Ein Auftrag der Firma Kümmerly \& Frey zur Berechnung einer Netzprojektion für eine Karte von Europa zwischen $30^{\circ}$ und $70^{\circ}$ Breite führte mich dazu, für die gewählte «Albers flächentreue Kegelprojektion» mit den längentreuen Breitenkreisen von $45^{\circ}$ und $62^{\circ}$ die Aequideformaten zu bestimmen.

Das Längenverzerrungsverhältnis kann für jede Kartenprojektion am einfachsten durch Linien gleicher Verzerrung, Aequideformaten geriannt, dargestellt werden. Diese Linien geben das Maß und den Verlauf der Längenverzerrung für das ganze Gebiet, über das die Projektion sich ausdehnt. Für die eingangs erwähnte Albers-Projektion soll als praktisches Beispiel die Ableitung und Darstellung von Aequideformaten gezeigt werden.

Bei jeder flächentreuen Kartenprojektion ist das Verzerrungsverhältnis, wie es in Tissots Verzerrungsellipse dargestellt wird, für die beiden Ellipsenachsen reziprok. Es gilt demnach für die Maximalverzerrung $h$ in der Meridianrichtung und der senkrecht dazu verlaufenden maximalen Verzerrung $\mathrm{k}$ im Breitenkreis die Gleichung

$$
\mathrm{h} \times \mathrm{k}=1 \text { oder } \mathrm{h}=\frac{1}{\mathrm{k}}
$$

Für die beiden längentreuen Breitenkreise $45^{\circ}$ und $62^{\circ}$ ist das Verzerrungsverhältnis $\mathrm{h}=\mathrm{k}=1$. Eine Längenvergrößerung tritt ein, wenn das Verhältnis größer als 1 ist, und eine Verkleinerung, wenn es als echter Bruch unter den Wert 1 sinkt. Für unser Beispiel tritt zwischen den beiden längentreuen Breitenkreisen in der Breitenkreisrichtung eine Verkleinerung und in der Meridianrichtung eine Vergrößerung ein. Diese Verzerrung erreicht ihr Maximum bei $54^{\circ} 22^{\prime}$ mit

$$
\begin{aligned}
& \mathrm{h}=1.011 \text { in der Meridianrichtung, und } \\
& \mathrm{k}=0.989016 \text { in der Breitenkreisrichtung }
\end{aligned}
$$

Außerhalb dieser Zone nehmen die Verzerrungen mit dem Abstand von den verzerrungsfreien Breitenkreisen zu und zwar um so rascher, je größer der Abstand wird. Doch handelt es sich nun um die reziproken Werte, in der Breitenkreisrichtung wird vergrößert, in der Meridianrichtung verkleinert. Dann nimmt die Verzerrung nördlich von $62^{\circ}$ bedeutend rascher zu als südlich von $45^{\circ}$, was für die gleiche Verzerrung von 1.07 folgendes Bild zeigt:

$\begin{array}{ccc}\text { Verzerrung } & \text { Breitenkreis } & \text { Breitenabstand } \\ 1.07 & 71^{0} 07^{\prime} & 9^{0} 07^{\prime} \\ 1.00 & 62^{0} 00^{\prime} & \\ 1.00 & 45^{\circ} 00^{\prime} & 18^{\circ} 44^{\prime} \\ 1.07 & 26^{\circ} 16^{\prime} & \end{array}$

